\title{
Medical Image of the Month: Thymolipoma
}

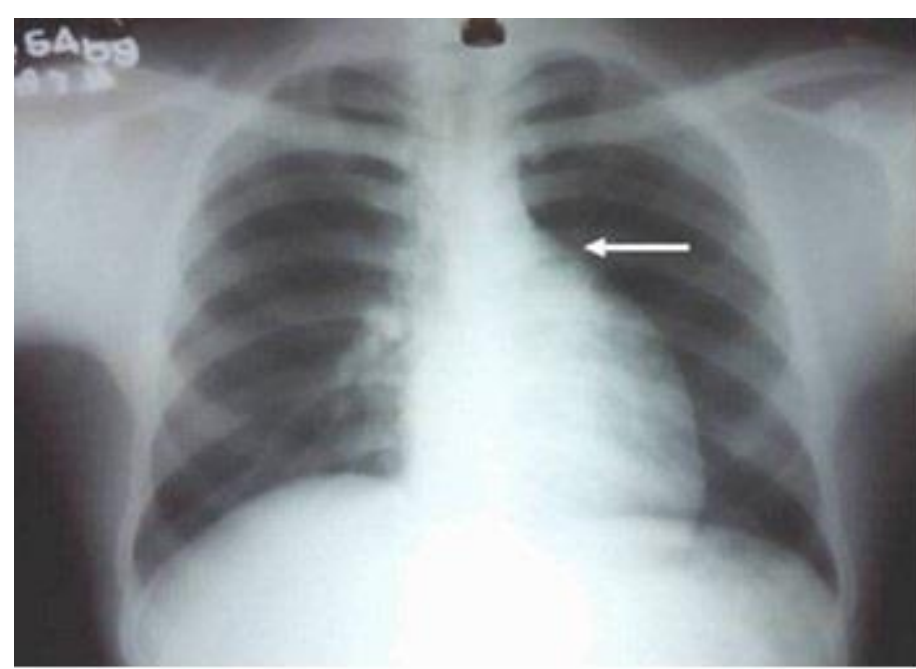

Figure 1. Chest radiograph PAview revealing subtle prominence of left upper heart border (white arrow).

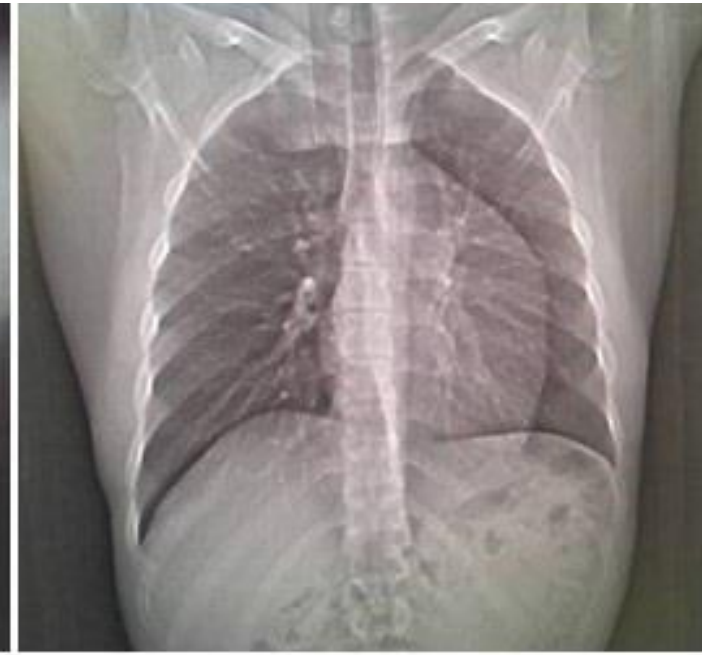

Figure 2. CT topogram: The

Prominence of the left heart is discretely seen suggesting pliability of the lesion (due to supine position).

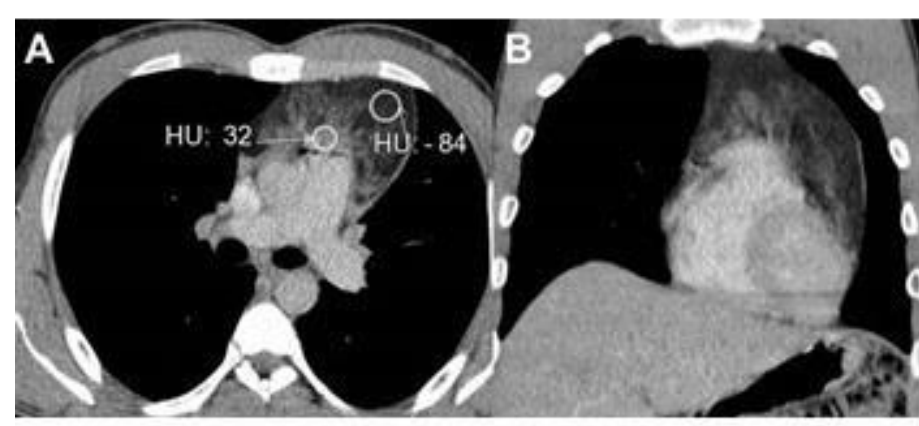

Figure 3. CECT axial and coronal images revealing fat containing anterior mediastinal mass with strands of soft tissues (HU values as depicted in image).

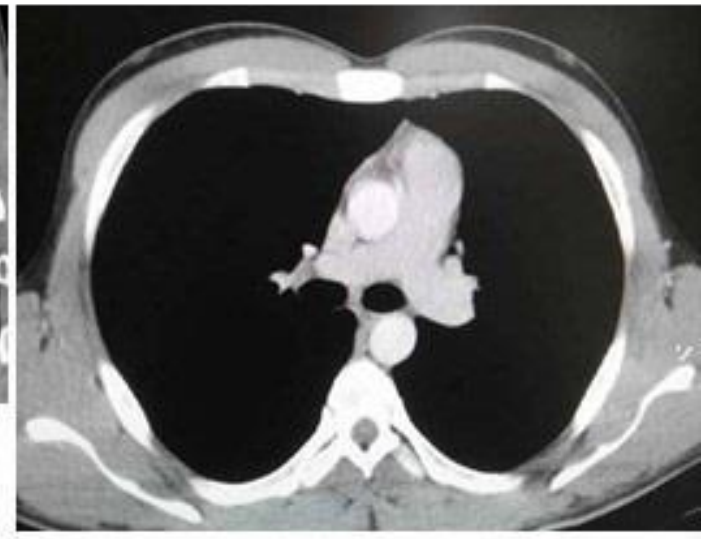

Figure 4. CECT of the thorax one year after resection: post contrast image revealing no mediastinal mass lesion

A 20-year-old man presented with a continuous, mild, dull aching pain affecting the left chest pain for 15 days duration. There were no aggravating or relieving factors nor any history of fever, respiratory or cardiac symptoms. The patient's vital signs were normal and examination of the respiratory and the cardiovascular systems revealed no abnormality. Electrocardiography and biochemical investigations, including cardiac enzymes, were normal. Chest radiography (Figure 1) revealed a subtle opacity causing fullness of the aorto-pulmonary window with subtle, smooth prominence of the left upper 
cardiac border; the left hilum was visualised discretely through the opacity (Figure 2). The patient underwent enhanced contrast chest CT (CECT, Figure 3) for further evaluation, revealing a large mass measuring $13 \times 7.9 \times 5 \mathrm{~cm}$ in the anterior mediastinum, extending from thoracic inlet to the left cardiophrenic angle. The mass consisted predominantly of fat density, with an average attenuation of - 84 Hounsfield units $(\mathrm{HU})$. Non-enhancing strands of soft tissue foci (mean, $32 \mathrm{HU}$ ) were also seen within the lesion. No areas of calcification or cystic degeneration were present. Planes between the subjacent mediastinal structures were preserved (Figures 2 and 3). A radiological impression of thymolipoma was offered. The patient was transferred to a tertiary care center where he underwent thoracoscopic excision and histopathology confirmed thymolipoma. Following surgery, the patient recovered uneventfully and follow up chest radiography and enhanced contrast chest CT (Figure 4) showed no evidence of lesion recurrence.

Thymolipoma is a rare benign tumor originating in the anterior mediastinum, and comprises $2-9 \%$ of all thymic neoplasms (1). Cytogenetic analyses have demonstrated that thymolipoma is a neoplasm of thymic fat (2). There is no sex or age predilection and affected patients range in age from 3-76 years. Most patients remain asymptomatic until the size of the lesion produced local mass effects, resulting in symptoms such as cough, dyspnea, hemoptysis, chest pain and hoarseness $(1,3)$. Autoimmune diseases, including myasthenia gravis, systemic lupus erythematosus, hypogammaglobulinemia, Graves' disease, and erythroblastopenia, may coexist in 10\% patients (1).

When thymolipomas are small, the lesion may not be detectable at chest radiography. When the lesion grows larger, it classically usually "drapes" around the heart and may simulate cardiomegaly (4). Extremely large thymolipomas have been described and can mimic pericardial effusion, pericardial cysts or pericardial tumors (5). Ultrasound can be used to differentiate fluid-containing lesions, such as pericardial effusion and cysts, from the echogenic fat typical of thymolipoma (4). Excessive epicardial fat, diaphragmatic elevation, sequestration or lobar collapse may also mimic this condition at chest radiography, whereas $\mathrm{CT}$ and/or MRI are diagnostic, revealing circumscribed anterior mediastinal fatty mass containing islands and strands of soft tissues with no invasion of adjacent structures (3-5).

Important differential diagnostic considerations for thymolipoma at cross sectional imaging include other fat-containing mediastinal masses such as mediastinal lipoma, mediastinal lipomatosis, liposarcoma and lipoblastoma. Lipomas are encapsulated whereas liopmatosis is an unencapsulated deposition of adipose tissues, and both lesions typically show homogeneous fat attenuation with no soft tissue strands.

Liposarcoma frequently occurs in the posterior mediastinum and is usually symptomatic at the time of presentation, and often manifests with inhomogeneous appearance and invasion of subjacent mediastinal structures on CT/ or MRI (6). Lipoblastoma usually occurs under the age of 3 , and shows intratumoral soft tissue stranding. Thymomas do not contain fatty tissue whereas some germ cell neoplasms contain cystic areas and calcification in addition to the fatty tissue $(3,6)$. CT is considered the modality of choice for the evaluation of mediastinal masses in general, and thymolipoma in particular. For 
patients in whom CT findings are equivocal, or for patients with contraindications to enhanced CT (such as contrast media allergy) MRI may be utilized for further characterization of mediastinal lesions, such as thymolipoma. Encasement or invasion of the mediastinal vasculature, esophagus, and trachea as well as involvement of the pericardium, myocardium, and pleura are accurately detected with MRI. The primary disadvantages of using MRI for mediastinal lesion characterization include limited identification of calcifications, longer imaging time, and higher cost $(3,6)$. Since thymolipomas are benign, and typically well encapsulated, with no invasion of surrounding structures, if detected incidentally, the lesion can be followed with imaging. However, when symptoms related to local mass effect develop, surgical resection is the treatment of choice which can be performed using a minimally invasive approach, such as thoracoscopic excision, with open resection reserved for larger lesions (7). Histopathological examination of thymolipoma reveals mature adipose tissue and hyperplastic thymic structures with Hassall's corpuscles (2).

\section{Amit Kumar Paliwal MD', Dr. Pradeep Jaiswal MCH (CTVS) AH (R\&R) ${ }^{2}$, and Dr. Vivek Sharma MD}

${ }^{1}$ Military Hospital Dehradun, Dehradun, Uttrakhand, India

${ }^{2}$ Delhi Cantt, Delhi, India

${ }^{3}$ Command Hospital (CC), Lucknow, India

\section{References}

1. Dongel I, Imamoglu H, Şahin AF, Yıldırım S, Bayram M. A rare mediastinal tumor: thymolipoma. Eur J Gen Med. 2014;11:21-3. [CrossRef]

2. Hudacko R, Aviv H, Langenfeld J, Fyfe B. Thymolipoma: Clues to pathogenesis revealed by cytogenetics. Ann Diagn Pathol. 2009;13:185-8.[CrossRef] [PubMed]

3. Tomiyama $\mathrm{N}$, Honda $\mathrm{O}$, Tsubamoto $\mathrm{M}$ et al. Anterior mediastinal tumors: diagnostic accuracy of CT and MRI. Eur J Radiol. 2009;69(2):280-8. [CrossRef] [PubMed]

4. Yeh HC, Gordon A, Kirschner PA, Cohen BA. Computed tomography and sonography of thymolipoma. AJR Am J Roentgenol. 1983;140(6):1131-3. [CrossRef] [PubMed]

5. Gamanagatti S, Sharma R, Hatimota $P$, Guleria R, Arvind S. Giant thymolipoma. AJR Am J Roentgenol. 2005;185(1):283-4. [CrossRef] [PubMed]

6. Juanpere S, Canete N, Ortuno P, Martínez S, Sanchez, G, Bernado L. A diagnostic approach to the mediastinal masses. Insights Imaging. 2013;4(1):29-52. [CrossRef] [PubMed]

7. Carapinha CP, Wainwright L, Loveland JA. A giant thymolipoma. S Afr J Child Health. 2010;4(1):20-1. 